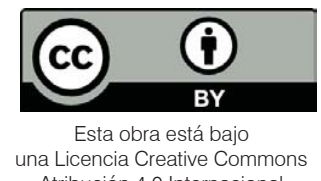

\title{
Características clínico - epidemiológicas y necesidad de tratamiento de los pacientes atendidos en el Servicio de Estomatología de Pacientes Especiales en un periodo de 2 años
}

Clinical and epidemiological features and dental care need of patients attended at Estomatología de Pacientes Especiales in a period of 2 years

José Antonio García Murga 1,2,a , Lillie Abanto Silva ${ }^{2, a}$

\section{RESUMEN}

Objetivo: Determinar las características clínico - epidemiológicas y necesidad de tratamiento de los pacientes especiales que recibieron atención en el Servicio de Estomatología de Pacientes Especiales de la Universidad Peruana Cayetano Heredia en el periodo de abril del 2016 a marzo de 2018. Material y Métodos: Se analizaron las variables procedencia, género, edad, diagnóstico sistémico y necesidad de tratamiento mediante estadística descriptiva. Resultados: La muestra estuvo conformada por 1735 historias clínicas que cumplieron los criterios de inclusión. El 87,9\% de pacientes viven en Lima Metropolitana y el 63 \% proviene de Lima norte. El 62,2\% fue de género femenino y el $37,8 \%$ de género masculino. La edad promedio de atención fue de $52,97 \pm 25,92$ años y el 50,26\% fueron mayores de 60 años. Se obtuvo una media de 1,97 $\pm 1,21$ diagnósticos sistémicos, con mayor prevalencia de las enfermedades que afectan al aparato circulatorio $(35,3 \%$ y las enfermedades endocrinas, metabólicas y nutricionales $(26,4 \%)$. En cuanto a necesidad de tratamiento, destacaron la operatoria dental (75\%), la rehabilitación oral $(59,4 \%)$, y el tratamiento quirúrgico (36,7\%). Conclusiones: La mayoría de la población especial atendida provino de la zona norte de Lima metropolitana, fue de género femenino y perteneció al grupo del adulto mayor. Además, las enfermedades más prevalentes fueron la hipertensión arterial $(29,51 \%)$ y la diabetes mellitus $(14,98 \%)$, necesitando principalmente tratamiento de operatoria dental y de rehabilitación oral.

PALABRAS CLAVE: Atención dental para personas con discapacidades, atención dental para enfermos crónicos, epidemiología.

\section{SUMMARY}

Objective: Determine the clinical and epidemiological characteristics and need for dental care in the population of special patients that received attention in EPE - CEC - UPCH since April from 2016 to March 2018. Material and Methods: We analyze variables, such as the origin, gender, age, systemic diagnoses and need for treatment through descriptive statistics. Results: The sample were 1735 clinical files that met the inclusion criteria. The $87.9 \%$ live in Lima city and $63 \%$ in the northern zone, $62.2 \%$ were female and $37.8 \%$ male. The average age was $52.97 \pm 25.92$ years old, and $50.26 \%$ were older than 60 years. Special patient has $1.97 \pm 1.21$ systemic diagnoses, with a higher prevalence of circulatory system diseases with $35.5 \%$ followed by endocrine, metabolic and nutritional diseases with $26.4 \%$. Regarding the need for dental care, the main treatments were dental restorations (75\%), oral rehabilitation (59.4\%), and dental surgery (36.7\%). Conclusions: Most part of the special population attended, came from the northern zone of Lima city, was female gender and belonged to the older adult group. In addition, the most prevalent diseases were arterial hypertension (29.51\%) and diabetes mellitus (14.98\%); and the main treatments were dental restorations and oral rehabilitation.

KEY WORDS: Dental care for disabled, dental care for chronically ill, epidemiology.

\footnotetext{
Facultad de Odontología, Universidad Nacional Mayor de San Marcos. Lima, Perú.

Facultad de Estomatología, Universidad Peruana Cayetano Heredia. Lima, Perú.

a Especialista en Estomatología de Pacientes Especiales,
} 


\section{INTRODUCCIÓN}

Un paciente especial es aquel que presenta síntomas y signos que lo alejan de la normalidad, que puede estar médicamente comprometido, o que pueda tener una condición especial física, psíquica, sensorial o trastornos del comportamiento (1).

Debemos considerar la posible relación entre el tratamiento dental y la enfermedad presente, evaluar la dificultad del manejo (tanto desde el punto de vista de conducta como de la técnica odontológica), y por supuesto, las lesiones relacionadas con el tratamiento médico recibido, como podrían ser las derivadas por radioterapia o por los medicamentos prescritos (2).

En esta categoría, también se incluyen enfermedades o condiciones que afectan solamente el complejo orofacial, como por ejemplo la dentinogénesis imperfecta, amelogénesis imperfecta, cáncer bucal o fisura labio alveolo palatina $(3,4)$.

En el Perú, el 10,4\% de la población presenta alguna discapacidad física y/o mental. Afecta con mayor prevalencia a los mayores de 60 años $(40,52 \%)$, seguido de los de 30 a 59 años $(34,69 \%)$, de 0 a 17 años $(14,34 \%)$ y de 18 a 29 años $(10,45 \%)$. Existe una mayor prevalencia en las mujeres $(57 \%)$ que en los hombres (43\%). De toda la población que presenta alguna discapacidad, el $81,5 \%$ tiene solo una y el $18,5 \%$ tiene dos o más (5).

La incidencia de enfermedades crónicas se incrementa con la edad. En los Estados Unidos, en el segmento de mayores de 65 años, casi un cuarto de ellos, toma uno o dos medicamentos prescritos, y casi cuatro de cada diez personas $(36,7 \%)$ toman cinco o más medicamentos. Por eso es importante entender, cómo el diagnóstico y el plan de tratamiento médico se interrelacionan con el diagnóstico y tratamiento dental $(6,7,8,9)$.

Los fármacos prescritos por el médico tratante, pueden interferir con el tratamiento estomatológico o pueden provocar alteraciones en la cavidad bucal, tal es el caso del tratamiento con anticoagulantes o con bifosfonatos, por ejemplo (10).

Son cuatro las mayores causas de muerte por enfermedades no trasmisibles (ENT): las afecciones cardiovasculares, cáncer, enfermedad pulmonar obstructiva crónica, y diabetes que corresponden al $82 \%$ del total. En el Perú, la probabilidad de morir de una de estas cuatro principales ENT entre los 30 y 70 años se estima en un $11,2 \%(11,12,13)$.

Las ENT evolucionan lentamente y no se trasmiten de persona a persona. Afectan a todos los grupos etarios y constituyen un problema serio de salud pública. En el 2016, el 8,6\% de la población mayor de 15 años presentó hipertensión arterial, mientras que el $66 \%$ de ellos recibieron y/o adquirieron medicamentos antihipertensivos. En ese mismo año, el 2,9\% de los mayores de 15 años debutaron con diabetes mellitus, y el $70 \%$ refirió recibir tratamiento hipoglucemiante (14). Las ENT influyen en el manejo odontológico de estos pacientes. Por ejemplo, las personas con diabetes presentan una mayor predisposición a padecer enfer- medad periodontal severa, con mayor tendencia a la hemorragia, pérdida de inserción y pérdida de soporte óseo; además, la hiperglucemia provoca alteraciones a nivel vascular e inmunológico que en conjunto favorecen a la progresión de infecciones a ese nivel. El paciente con cardiopatía congénita puede requerir profilaxis antibiótica para evitar la endocarditis infecciosa que puede ser provocada por un tratamiento dental de rutina. De esta misma manera, los pacientes con insuficiencia renal crónica, hipertensión arterial, hipotiroidismo, etc., presentarán condiciones estomatológicas que requerirán terapias adecuadas según sea el caso. Estas condiciones médicas representan un alto riesgo sobre el sistema estomatognático, presentándose con mayor frecuencia la enfermedad periodontal, las maloclusiones dentales y la caries dental $(10,15,16)$.

El objetivo del presente trabajo fue determinar las características clínico - epidemiológicas y la necesidad de tratamiento de los pacientes atendidos en el Servicio de Estomatología de Pacientes Especiales de la Universidad Peruana Cayetano Heredia en en el periodo de abril de 2016 a marzo del 2018.

\section{MATERIAL Y MÉTODOS}

El presente trabajo es un estudio transversal, observacional, descriptivo y retrospectivo. La población estuvo conformada por las historias clínicas digitales de todos los pacientes atendidos en el Servicio de Estomatología de Pacientes Especiales de la Universidad

Peruana Cayetano Heredia en durante el periodo del 01 de abril de 2016 hasta el 31 de marzo del 2018, haciendo un total de 1840 .

La muestra estuvo constituida por historias clínicas digitales que cumplieron con todos los criterios de inclusión (contar con los registros de edad, género, lugar de procedencia, diagnóstico sistémico, medicación, motivo de consulta, diagnóstico estomatológico, plan de tratamiento durante el periodo estudiado y no estar duplicada) y se excluyeron las que no los cumplieron, resultando 1735 historias válidas donde se estudiaron las variables procedencia, género, edad, diagnóstico sistémico y necesidad de tratamiento.

Se gestionó el permiso correspondiente en la CEC $\mathrm{UPCH}$, se revisó y se registró la información en una ficha de recolección de datos. Este estudio fue realizado luego de recibir la aprobación de la Unidad Integrada de Gestión, Investigación, Ciencia y Tecnología de las Facultades de Medicina, Estomatología y de Enfermería y la posterior aprobación del Comité Institucional de Ética de la Universidad Peruana Cayetano Heredia (CIE - UPCH) según Constancia 334-14-18.

El procesamiento de datos se trabajó mediante un 
análisis de estadística descriptiva de las variables con la obtención de sus frecuencias absolutas y relativas empleando el software estadístico IBM SPSS v. 25.0.

\section{RESULTADOS}

El mayor número de pacientes se registró procedentes de Lima con 87,95\% (1 526 pacientes), Callao 6,29\% (109 pacientes) y Provincias 5,76\% (100 pacientes), principalmente de Lima Norte $(63 \%)$; y los distritos de mayor afluencia fueron San Martín de Porres 29,3\% (447), Los Olivos 10,7\% (164 pacientes), y Comas 9\% (138 pacientes). Existe un predominio de la población femenina con $62,2 \%$ (1 079 pacientes) sobre la masculina con $37,8 \%$ (656 pacientes). Se evidencia que el promedio de edad fue $52,97 \pm 25,92$ años. Las décadas más prevalentes fueron de 70 a 79 años 18,7\% (325 pacientes), 60 a 69 años 16,7\% (289 pacientes), y de 80 a 89 años 12,3\% (214 pacientes) (tabla 1). En la tabla 2 se muestra que el promedio de diagnósticos sistémicos fue de 1,97 $\pm 1,21$ con un mínimo de cero diagnósticos, correspondiente al paciente adulto mayor sano $2,5 \%$ (43 pacientes) y un máximo de ocho diagnósticos sistémicos $0,1 \%$ (1 pacientes), siendo los más prevalentes los pacientes con uno $41,2 \%$ (715 pacientes), dos 30,5\% (530 pacientes), y de tres a más diagnósticos $25,76 \%$ (447 pacientes). Las enfermedades más prevalentes fueron las del aparato circulatorio 35,3\% (612 pacientes); las enfermedades endocrinas, metabólicas y nutricionales $26,4 \%$ (458 pacientes); y los trastornos mentales y de comportamiento $18 \%$ (312 pacientes) (tabla 3 ).

Tabla 1. Características demográficas de los pacientes atendidos en EPE - CEC - UPCH en el periodo de abril de 2016 a marzo de 2018.

\begin{tabular}{|c|c|c|c|}
\hline \multicolumn{4}{|c|}{ Características Demográficas } \\
\hline \multirow{2}{*}{ Variables } & & \multicolumn{2}{|c|}{ Pacientes n: 1735} \\
\hline & & $\mathrm{n}$ & $\%$ \\
\hline \multirow[t]{3}{*}{ Procedencia } & Provincia Constitucional del Callao & 109 & 6.29 \\
\hline & Lima Metropolitana & 1526 & 87.95 \\
\hline & Provincias & 100 & 5.76 \\
\hline \multirow[t]{2}{*}{ Género } & Femenino & 1079 & 62.19 \\
\hline & Masculino & 656 & 37.81 \\
\hline Edad & $0-9$ años & 154 & 8.88 \\
\hline \multirow[t]{10}{*}{$\bar{x}: 52.97$ años } & $10-19$ años & 102 & 5.88 \\
\hline & $20-29$ años & 135 & 7.78 \\
\hline & $30-39$ años & 134 & 7.72 \\
\hline & $40-49$ años & 135 & 7.78 \\
\hline & $50-59$ años & 203 & 11.70 \\
\hline & $60-69$ años & 289 & 16.66 \\
\hline & $70-79$ años & 325 & 18.73 \\
\hline & $80-89$ años & 214 & 12.33 \\
\hline & $90-99$ años & 43 & 2.48 \\
\hline & 100 años a más & 1 & 0.06 \\
\hline
\end{tabular}


Tabla 2. Distribución de pacientes atendidos en EPE - CEC - UPCH, según el número de diagnósticos sistémicos, en el periodo de abril de 2016 a marzo de 2018

\begin{tabular}{lrr}
\hline \multicolumn{3}{c}{ Número de Diagnósticos sistémicos } \\
& Frecuencia & \multicolumn{1}{c}{ Porcentaje } \\
\hline 0 & 43 & $2.48 \%$ \\
1 & 715 & $41.21 \%$ \\
2 & 530 & $30.55 \%$ \\
3 & 251 & $14.47 \%$ \\
4 & 121 & $6.97 \%$ \\
5 & 49 & $2.82 \%$ \\
6 & 18 & $1.04 \%$ \\
7 & 7 & $0.40 \%$ \\
8 & 1 & $0.06 \%$ \\
Total & 1735 & $100.00 \%$ \\
\hline
\end{tabular}

${ }^{\dagger}$ Promedio: 1.97

Tabla 3. Prevalencia de diagnósticos sistémicos por grupos de enfermedades según CIE - 10 de los pacientes atendidos en EPE - CEC - UPCH, en el periodo de abril de 2016 a marzo de 2018.

n: 1735

Diagnósticos Sistémicos

\begin{tabular}{|c|c|c|}
\hline Grupos de Enfermedades sistémicas & Frecuencia & Porcentaje \\
\hline I. Ciertas enfermedades infecciosas y parasitarias & 79 & $4.6 \%$ \\
\hline II. Neoplasias & 126 & $7.3 \%$ \\
\hline III. Enfermedades de la sangre, órganos hematopoyéticos y del mecanismo inmunológico & 31 & $1.8 \%$ \\
\hline IV. Enfermedades endocrinas, nutricionales y metabólicas & 458 & $26.4 \%$ \\
\hline V. Trastornos mentales y de comportamiento & 312 & $18.0 \%$ \\
\hline VI. Enfermedades del Sistema nervioso & 230 & $13.3 \%$ \\
\hline VII. Enfermedades del ojo y sus anexos & 83 & $4.8 \%$ \\
\hline VIII. Enfermedades del oído y de la apófisis mastoide & 30 & $1.7 \%$ \\
\hline IX. Enfermedades del aparato circulatorio & 612 & $35.3 \%$ \\
\hline X. Enfermedades del aparato respiratorio & 102 & $5.9 \%$ \\
\hline XI. Enfermedades del aparato digestivo & 137 & $7.9 \%$ \\
\hline XII. Enfermedades de la piel y del tejido subcutáneo & 30 & $1.7 \%$ \\
\hline XIII. Enfermedades del aparato musculoesquelético y del tejido conectivo & 302 & $17.4 \%$ \\
\hline XIV. Enfermedades del aparato génitourinario & 103 & $5.9 \%$ \\
\hline XV. Malformaciones congénitas, deformidades y anomalías cromosómicas & 209 & $12.0 \%$ \\
\hline XVI. Factores que influyen en el estado de salud & 87 & $5.0 \%$ \\
\hline XVII. Síntomas, signos y hallazgos clínicos o de laboratorio anormales & 43 & $2.5 \%$ \\
\hline XVIII. Heridas, envenenamiento y otras consecuencias de causas externas & 6 & $0.3 \%$ \\
\hline XIX. Condiciones originadas en el periodo perinatal & 2 & $0.1 \%$ \\
\hline
\end{tabular}


La enfermedad del aparato circulatorio más prevalente fue la hipertensión arterial $83,7 \%$ (512 pacientes), mientras que la enfermedad endocrina, nutricional y metabólica más prevalente fue la diabetes mellitus
$56,8 \%$, y además se aprecia que el trastorno mental y de comportamiento más prevalente fue el retardo mental 35,6\% (111 pacientes) como se detalla en la tabla 4, 5, y 6 respectivamente.

Tabla 4. Prevalencia de las enfermedades del aparato circulatorio en los pacientes atendidos en EPE - CEC - UPCH en el periodo de abril de 2016 a marzo de 2018.

\begin{tabular}{|c|c|c|c|}
\hline \multicolumn{4}{|c|}{ Enfermedades del aparato circulatorio } \\
\hline & & Frecuencia & Porcentaje \\
\hline \multirow[t]{10}{*}{$\mathrm{N}: 612$} & Hipertensión arterial & 512 & $83.7 \%$ \\
\hline & Arritmia & 63 & $10.3 \%$ \\
\hline & Accidente cerebrovascular & 53 & $8.7 \%$ \\
\hline & Insuficiencia Venosa & 28 & $4.6 \%$ \\
\hline & Ateroesclerosis & 17 & $2.8 \%$ \\
\hline & Valvulopatías & 16 & $2.6 \%$ \\
\hline & Infarto al Miocardio Agudo & 15 & $2.5 \%$ \\
\hline & Angina de pecho & 14 & $2.3 \%$ \\
\hline & Trombosis & 5 & $0.8 \%$ \\
\hline & Otros & 35 & $5.7 \%$ \\
\hline
\end{tabular}

Tabla 5. Prevalencia de las enfermedades endocrinas, nutricionales y metabólicas en los pacientes atendidos en EPE - CEC - UPCH en el periodo de abril de 2016 a marzo de 2018.

Enfermedades endocrinas, nutricionales y metabólicas

\begin{tabular}{llrc} 
& Frecuencia & Porcentaje \\
\hline N: 458 & 260 & $56.8 \%$ \\
& Diabetes mellitus & 167 & $36.5 \%$ \\
Hipotiroidismo & 84 & $18.3 \%$ \\
Dislipidemia & 5 & $1.1 \%$ \\
Hipertiroidismo & 2 & $0.4 \%$ \\
Insuficiencia adrenal & 12 & $2.5 \%$ \\
Otros & \\
\hline
\end{tabular}


Tabla 6. Prevalencia de los trastornos mentales y del comportamiento en los pacientes atendidos en EPE CEC - UPCH en el periodo de abril de 2016 a marzo de 2018.

\begin{tabular}{llrc}
\hline \multicolumn{1}{c}{ Trastornos mentales y del comportamiento } & & \\
& & Frecuencia & Porcentaje \\
\hline N: 312 & Retardo mental & 111 & $35.6 \%$ \\
& Depresión & 73 & $23.4 \%$ \\
& Trastorno del espectro autista & 62 & $19.9 \%$ \\
& Ansiedad & 60 & $19.2 \%$ \\
& Esquizofrenia & 27 & $8.7 \%$ \\
Trastorno bipolar & 9 & $2.9 \%$ \\
& Demencia senil & 6 & $1.9 \%$ \\
Trastorno obsesivo compulsivo & 5 & $1.6 \%$ \\
Psicosis & 4 & $1.3 \%$ \\
Otros & 11 & $3.5 \%$ \\
\hline
\end{tabular}

En la tabla 7 se muestran los diez diagnósticos sistémicos más prevalentes que incluyen hipertensión arterial 29,51\% (512 pacientes), diabetes mellitus $14,98 \%$ (260 pacientes), e hipotiroidismo 9,62\% (167 pacientes). La necesidad de tratamiento estomato- lógico más prevalente fue la operatoria dental $75 \%$ (1302 pacientes), seguido de la rehabilitación oral $59,4 \%$ (1031 pacientes) y del tratamiento quirúrgico $36,7 \%$ (636 pacientes) (tabla 8 ).

Tabla 7. Diez diagnósticos sistémicos más prevalentes de los pacientes atendidos en EPE - CEC - UPCH, en el periodo de abril de 2016 a marzo de 2018.

\begin{tabular}{lcc}
\hline \multicolumn{2}{c}{ Diagnóstico sistémico } \\
Frecuencia & Porcentaje \\
\hline Hipertensión arterial & 512 & $29.51 \%$ \\
Diabetes mellitus & 260 & $14.98 \%$ \\
Hipotiroidismo & 167 & $9.62 \%$ \\
Osteoporosis & 150 & $8.64 \%$ \\
Fisura labiopalatina & 127 & $7.31 \%$ \\
Artrosis & 122 & $7.03 \%$ \\
Retardo mental & 111 & $6.39 \%$ \\
Dislipidemia & 84 & $4.84 \%$ \\
Gastritis & 81 & $4.66 \%$ \\
Epilepsia & 76 & $4.38 \%$ \\
\hline
\end{tabular}


Tabla 8. Necesidad de tratamiento estomatológico de los pacientes atendidos en EPE - CEC - UPCH, en el periodo de abril de 2016 a marzo de 2018.

\begin{tabular}{llrr}
\hline & \multicolumn{1}{c}{ Tipo de Tratamiento } & & \\
& & Frecuencia & Porcentaje \\
\hline N: 1735 & 14 & $0.8 \%$ \\
& Preventivo & 1302 & $75.0 \%$ \\
& Operatoria Dental & 198 & $11.4 \%$ \\
& Pulpar & 636 & $36.7 \%$ \\
Quirúrgico & 1031 & $59.4 \%$ \\
Rehabilitación Oral & 229 & $13.2 \%$ \\
Periodontal & 86 & $5.0 \%$ \\
\hline
\end{tabular}

\section{DISCUSIÓN}

Nuestra población de estudio se concentró en solo seis de los cuarenta y tres distritos de Lima metropolitana, particularmente de la zona norte (San Martín de Porres, Independencia, Los Olivos, Carabayllo, Comas y Puente Piedra) representado por un $61,8 \%$ de la población estudiada y dejando muy por detrás a otras zonas populosas de nuestra ciudad. Haciendo una comparación de los resultados obtenidos en el Censo Nacional 2017 (5), donde Lima Norte cuenta con 28,05\%, Lima Este 27,78\%, Lima Centro 23,11\% y Lima Sur $21,06 \%$, con nuestro estudio, que contó con Lima Norte 63\%, Lima Centro 23,2\%, Lima Este $11,3 \%$ y Lima Sur 2,5\% observamos una notable diferencia sobre todo en la procedencia de Lima Sur y Lima Este.

Las dos terceras partes de la población estudiada $(62,2 \%)$ fueron de género femenino mientras que el tercio restante $(37,8 \%)$ fueron de género masculino; resultados que son similares a los obtenidos en el Censo Nacional 2017 (5), el INEI en el 2016, 2015 y $2012(17,18,19)$, y a los estudios de Leyva (20), Hernández-Millán et al., (21) y Fernández-Feijoo et al., (22) donde encontraron que el género femenino prevaleció con un $57 \%, 50,3 \%, 52,1 \%, 52,1 \%, 59 \%$, $54,69 \%$, y $58,4 \%$, respectivamente.

La edad promedio de la población de pacientes especiales que se atiende en nuestro servicio fue 52,97 $\pm 25,92$ años, que es ligeramente mayor a las encontradas por Leyva (20) y Fernández-Feijoo et al., (22) cuyas edades promedio fueron $47,98 \pm 28,06$ años $y$ $45,7 \pm 17,5$ años respectivamente.
Los resultados del Censo Nacional 2017 (5) muestran la asociación entre edad y discapacidad, mostrando que a mayor edad, es mayor el porcentaje de personas que declaran tener alguna discapacidad, de tal manera que de 0 a 17 años se obtuvo $14,34 \%$, de 18 a 29 años de edad $10,45 \%, 30$ a 59 años $34,69 \%$ y de 60 a más años un 40,52\%; y en nuestro estudio se obtuvo la misma tendencia, de tal manera que de 0 a 19 años fueron $14,8 \%$, de 20 a 59 años $35 \%$, y mayores de 60 años fueron el 50,2\% y además, en grupos etarios por décadas obtuvimos la mayor prevalencia de pacientes de 70 - 79 años con $18,7 \%$, seguido del grupo de 60 69 años con $16,7 \%$ y en tercer lugar por el grupo de 80 a más años con $14,9 \%$. Estos últimos hallazgos coinciden con los datos obtenidos por la ENEDIS 2012 (18) que señalan las décadas más prevalentes de 70 a 79 años con $26,2 \%$, seguido de los de 60 a 69 años con $24,1 \%$, y en tercer lugar los de 80 años a más con $15,7 \%$; resultado similar al estudio de Leyva (20) que obtuvo de $70-79$ años $18,4 \%, 80$ a más años $17,7 \%$, y 60 a 69 con 15,3\%. Por el contrario, nuestros resultados difieren de lo encontrado por Hernández-Millán et al., (21), que obtuvo de 35 a 59 años 47,92\%, 60 a 74 años $19,79 \%$ y de 20 a 34 años $18,75 \%$.

De acuerdo al número de condiciones sistémicas que se presentan en la población de pacientes especiales, nuestro estudio arrojó una media de $1,97 \pm 1,21$, con una prevalencia de $41,2 \%$ para un diagnóstico, $30,5 \%$ para dos diagnósticos y $25,8 \%$ para tres diagnósticos a más; resultados similares a lo encontrado por Leyva (20), el Censo Nacional 2017 (5), y la ENEDIS 2012 (18) donde prevaleció la presencia de una limitación con $66 \%, 81,5 \%$, y $38,6 \%$ respectivamente. 
Nuestro estudio mostró una prevalencia mayor de las enfermedades del aparato circulatorio con un 35,3\%, seguido de las enfermedades endocrinas, nutricionales y metabólicas con $26,4 \%$, de los trastornos mentales y de comportamiento con $18 \%$, similar a lo encontrado por Leyva (20) con las enfermedades cardiovasculares $26,8 \%$, enfermedades endocrinas, nutricionales y metabólicas $16,4 \%$, trastornos mentales y de comportamiento $11,9 \%$; y de Fernández-Feijoo et al., (22), donde la enfermedad cardiovascular obtiene un $16,1 \%$ de prevalencia, y las enfermedades endocrinas y metabólicas un 13,7\%.

Por enfermedad específica, las que afectaron mayormente a nuestra población fueron la hipertensión arterial con $29,51 \%$, diabetes mellitus $14,98 \%$, e hipotiroidismo $9,62 \%$. Similares resultados se obtuvieron en la ENEDIS 2012 (18), con hipertensión arterial/ taquicardia $43,9 \%$, y diabetes/obesidad $21,8 \%$, y en el estudio de Leyva (20) con una mayor prevalencia de hipertensión arterial $27,7 \%$, y diabetes mellitus $15,6 \%$. Por el contrario, en el estudio de Hernández-Millán et al., (21), se encontró que lo más frecuente fue la diabetes mellitus $34,37 \%$, hipertensión arterial $29,65 \%$, y epilepsia $14,06 \%$.

En cuanto a la necesidad de tratamiento, en nuestro estudio resultó que la gran mayoría de los pacientes necesitaron tratamiento de operatoria dental $75 \%$, rehabilitación oral $59,4 \%$, quirúrgico $36,7 \%$, periodontal $13,2 \%$, pulpar $11,4 \%$, ortodoncia y ortopedia $5 \%$, y preventivo $0,8 \%$. Lo que difiere de lo encontrado por Hernández-Millán et al., (21), con tratamiento periodontal $65,1 \%$, operatoria dental $33,33 \%$, rehabilitación oral $32,29 \%$, quirúrgico $17,19 \%$, ortodoncia $5,21 \%$, y pulpar con $2,08 \%$, tal vez debido a que en su estudio encontró una gran prevalencia de pacientes con diabetes mellitus que está fuertemente asociada a la enfermedad periodontal. Hay que precisar también, que es probable que en nuestro estudio el tratamiento periodontal y el tratamiento preventivo no tengan un porcentaje importante ya que no se tomó en cuenta la profilaxis dental y la aplicación de flúor, salvo en los pacientes donde fue el único tratamiento realizado, ya que son procedimientos que normalmente se realizan a todos los pacientes del servicio antes de iniciar el plan de tratamiento.

En conclusión, más de la mitad de la población de pacientes especiales que se atendieron en el servicio de EPE - CEC - UPCH en el periodo estudiado provino del cono norte de Lima Metropolitana (63\%). Debido quizás, a que la sede central se encuentra en el distrito de San Martín de Porres, y a la distancia y difícil acceso desde los distritos más alejados. Esto nos brinda la oportunidad de abrir una sucursal en la sede de la Clínica Estomatológica de la UPCH en el distrito de San Isidro y poder captar una mayor afluencia de los pacientes que provengan del cono este y cono sur de nuestra ciudad, que claramente están más distanciados de nuestra cobertura de atención. Casi dos tercios de la población atendida fueron de género femenino con un $62,2 \%$, mientras que el masculino fue de $37,8 \%$. Ya que nuestra población estudiada fue predominantemente femenina, se debería tener especial consideración en el estudio de los estados fisiológicos y las enfermedades predominantes que afectan a este género como la gestación, la menopausia, osteoporosis, etc. La mitad de la población de pacientes especiales que se atendieron en nuestro servicio fueron adultos mayores $(50,2 \%)$ con una edad promedio de 52,97 años y un pico entre los $70-79$ años $(18,7 \%)$. Por ello, es necesario seguir mejorando la infraestructura del servicio como la instalación de pasamanos en el trayecto hacia las unidades, evitar ruidos excesivos, zonas más amplias para su desplazamiento, etc. Así como también, hacer énfasis en el reconocimiento de los signos y síntomas de las enfermedades o condiciones sistémicas que se presentan en este grupo etario como la hipertensión arterial, la diabetes mellitus, osteoporosis, demencia senil, anemia, etc. El paciente especial en promedio presenta 1,97 diagnósticos sistémicos, siendo los grupos de enfermedades más prevalentes las enfermedades del aparato circulatorio $(35,3 \%)$, seguido de las enfermedades endocrinas, metabólicas, y nutricionales $(26,4 \%)$, y los trastornos mentales y del comportamiento (18\%). Los diagnósticos sistémicos más prevalentes en la población estudiada fueron la hipertensión arterial con $29,51 \%$, la diabetes mellitus 14,98\%, el hipotiroidismo 9,62\%, y la osteoporosis $8,64 \%$. Con respecto a la necesidad de tratamiento, casi tres cuartos de la población necesitaron tratamiento de operatoria dental (75\%), casi dos tercios de rehabilitación oral $(59,4 \%)$, y un tercio del tratamiento quirúrgico $(36,7 \%)$.

Estos datos son importantes para que el especialista en Estomatología de Pacientes Especiales tenga un mejor conocimiento y manejo de las manifestaciones bucales que podrían presentarse en estas enfermedades y/o en el uso de su terapia farmacológica, así como también se capacite con mayor énfasis en los tratamientos que poseen la mayor demanda para esta población. 


\section{Correspondencia:}

\author{
José Antonio García Murga \\ Correo electrónico: jose.garcia.m@upch.pe
}

\section{REFERENCIAS BIBLIOGRÁFICAS}

1. Oliveira J, Sandoval S, Andrade F. Caracterización clínico - epidemiológica de pacientes especiales con maloclusión. Polo del Conocimiento. 2017;2(6):12981310.

2. Silvestre-Donat FJ, Silvestre-Rangil J, Espín-Gálvez F. La atención hospitalaria pública a los pacientes especiales. Medicina Oral, Patología Oral y Cirugía Bucal. 2014;19(3):163-169.

3. American Academy of Pediatric Dentistry. Management of Dental Patients with Special Health Care Needs. Chicago: American Academy of Pediatric Dentistry; 2016. (Citado el 10 de enero del 2020) Disponible en: http://www.aapd.org/media/Policies_Guidelines/BP_SHCN.pdf

4. Vozza I, Cavalle E, Corridore D, et al. Preventive strategies in oral health for special needs patients. Annali di Stomatologia. 2015;6(3-4):96-99.

5. Instituto Nacional de Estadística e Informática. Perú: Perfil Sociodemográfico Informe Nacional Censo 2017. Lima: Instituto Nacional de Estadística e Informática; 2018.

6. Little JW, Miller CS, Rhodus NL. Little and Falace's dental management of the medically compromised patient. St. Louis: Elsevier; 2017.

7. Ganda K. Dentist's guide to medical conditions, medications and complications. Ames: Wiley Blackwell; 2013.

8. CDC/National Center for Health Statistics. National Health Interview Survey. Maryland: National Center for Health Statistics; 2018. (Citado el 10 de enero del 2020) Disponible en: https://www.cdc.gov/nchs/health_policy/adult_chronic_conditions.htm

9. Ward BW, Schiller JS, Goodman RA. Multiple chronic conditions among US adults: a 2012 update. Prev Chronic Dis. 2014;11:130389.

10. García-Flores K, Suárez-Zafra D, Huerta-Flores K. Panorámica de la atención estomatológica a pacientes discapacitados o especiales. Revista de Ciencias Médicas La Habana. 2013;19(2):1-9.

11. World Health Organization. Global Status Report on noncommunicable diseases 2014. Geneva: World Health Organization Press; 2014.

12. Freid VM, Bernstein AB, Bush MA. Multiple chronic conditions among adults aged 45 and over: Trends over the Past 10 years. NCHS Data Brief. 2012;100:1-8.
13. nstituto Nacional de Estadística e Informática. Perú Encuesta Demográfica y de Salud Familiar 2016 Nacional y Regional. Lima: Instituto Nacional de Estadística e Informática; 2017.

14. Instituto Nacional de Estadística e Informática. Perú: Enfermedades no transmisibles y transmisibles, 2016. Lima: Instituto Nacional de Estadística e Informática; 2017.

15. Gu Q, Dillon CF, Burt VL. Prescription Drug use continues to increase: US Prescription drug data for 2007 - 2008. NCHS Data Brief. 2010;42:1-8.

16. Castellanos-Suárez JL, Díaz-Guzmán LM, Lee-Gómez EA. Medicina en Odontología Manejo dental de pacientes con enfermedades sistémicas. Ciudada de México: Manual Moderno; 2015.

17. Instituto Nacional de Estadística e Informática. Perú: Caracterización de las condiciones de vida de la población con discapacidad 2016. Lima: Instituto Nacional de Estadística e Informática; 2017.

18. Instituto Nacional de Estadística e Informática. Perú: Características de la Población con Discapacidad. Lima: Instituto Nacional de Estadística e Informática; 2015.

19. Instituto Nacional de Estadística e Informática. Primera Encuesta Nacional Especializada sobre Discapacidad 2012. Lima: Instituto Nacional de Estadística e Informática; 2014.

20. Leyva H. Identificación de enfermedades sistémicas y condiciones discapacitantes en pacientes atendidos en el servicio de estomatología de pacientes especiales de la Facultad de Estomatología "Roberto Beltrán" entre los años 2015 - 2016. Tesis para obtener el título de Cirujano Dentista. Lima: Universidad Peruana Cayetano Heredia; 2016.

21. Hernández-Millán A, Sueiro-Sánchez I, Manresa -Peñalver C, Hernández-Millán Z, Hurtado-Sánchez D, Barrio-Pedraza T. Determinación del estado de salud bucal en pacientes discapacitados del área VII en Cienfuegos. Revista Finlay. 2014;4(3):1-10.

22. Fernández-Feijoo J, Garea-Gorís R, Fernández-Varela M, Tomás-Carmona I, Diniz-Freitas M, Limeres-Posse J. Prevalence of systemic diseases among patients requesting dental consultation in the public and private systems. Med Oral Patol Oral Cir Bucal. 2012;17(1):89-93.

Recibido: 16-09-2019

Aceptado: 07-12-2019 